\section{MMW-Arzneimittelpreis 2013 für Sitagliptin}

_ Der seit 2007 in Deutschland verfügbare DPP-4-Inhibitor Sitagliptin (Januvia ${ }^{\circledR}$ ) hat den MMW-Arzneimittelpreis 2013 erhalten. Sitagliptin ist zur Therapie von Patienten mit Diabetes mellitus Typ 2 in Deutschland seit März 2007 zugelassen, und zwar in Mono- sowie in Kombinationstherapie. Seit Dezember 2011 kann der DPP-4-Hemmer auch bei Patienten mit Niereninsuffizienz in allen Stadien verordnet werden. Der ausgezeichnete Wirkstoff wurde also, so betonte MMW-Schriftleiter Prof. Hermann S. FüeßI durch therapiebegleitende Forschung entscheidend weiterentwickelt.

Prof. Hellmut Mehnert, München, bezeichnete DPP-4-Hemmer als das wichtigstes Bindeglied zwischen Forschung und praktischer Anwendung inkretinbasierter Antidiabetika, deren Entwicklung auf den wegweisenden Arbeiten der deutschen Arbeitsgruppe um Creutzfeld beruht. Gliptine hemmen das Enzym Dipeptidylpeptidase-4 (DPP-4) und verzögern dadurch den Abbau der Inkretinhormone GLP-1 (Glucagon-ähnliches Peptid-1) und GIP (Glukoseabhängiges insulinotropes Polypeptid). Bei Patienten mit Diabetes mellitus Typ 2 verlängern und verstärken sie damit die glukoseabhängige Wirkung der Inkretinhormone. Damit wirken DPP-4-Hemmer nur bei erhöhtem Blutzucker. „Es kommt nicht zu Hypoglykämien, weil es sich um intelligente Substanzen handelt", erläuterte Mehnert weiter. Darüber hinaus verringert Sitagliptin in Abhängigkeit von der Glukosekonzentration die Glukagonsekretion aus den Alphazellen der Bauchspeicheldrüse und vermindert dadurch die Glukosefreisetzung aus der Leber. Bei Patienten mit Diabetes mellitus Typ 2 und Hyperglykämie sinken bei Behandlung mit Sitagliptin die $\mathrm{HbA}_{1 c}$-Spiegel, die Nüchternglukosewerte und die postprandialen Glukosekonzentrationen. Ein weiterer Vorteil ist, dass mit Sitagliptin das Körpergewicht der Patienten eher abnimmt, während es bei Behandlung mit Sulfonyl-

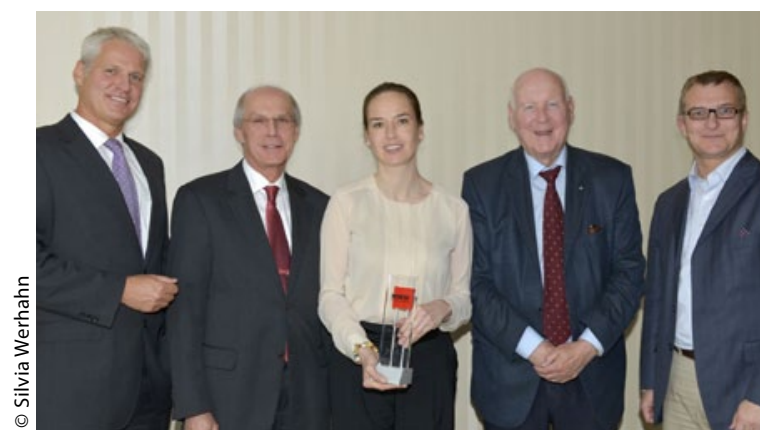

Dr. Dirk Einecke, MMW-Chefredakteur, Prof. Hermann S. FüeßI, Schriftleiter der MMW, Stefanie Tübben, MSD Sharp \& Dohme, Prof. Hellmut Mehnert, München, Dr. Elmar Jäckel, Hannover

harnstoffen steigt. Möglicherweise hat Sitagliptin auch kardioprotektive Effekte. Erste Langzeitergebnisse dazu werden im nächsten Jahr erwartet.

Dr. Susanne Heinzl

Verleihung des MMW-Arzneimittelpreises, Ulm, 6.7.2013

\title{
Welches Antiarrhythmikum für welchen Patienten?
}

_ Für symptomatische Patienten mit paroxysmalem oder persistierendem Vorhofflimmern ist die Stabilisierung des Sinusrhythmus ein wichtiges Therapieziel. „Die Wahl des geeigneten Antiarrhythmikums orientiert sich primär daran, ob eine strukturelle Herzerkrankung vorliegt oder nicht", so Prof. Stephan Hohnloser von der Kardiologischen Universitätsklinik in Frankfurt/ Main. Bei Patienten ohne bzw. mit einer moderaten strukturellen Herzerkrankung können Dronedaron (Multaq ${ }^{\circledast}$ ), Flecainid, Propafenon und Sotalol eingesetzt werden. Sollte diese Medikation nicht ausreichen, kann die medikamentöse Therapie entweder auf Amiodaron umgestellt oder eine Katheterablation durchgeführt werden.

Doch bei Patienten mit struktureller Herzerkrankung sind Klasse-I-Antiarrhythmika wie Flecainid und Propafenon kontraindiziert. Bei einer hypertensiven Herzerkrankung mit linksventrikulärer Hypertrophie empfiehlt sich als Erstlinien-Therapie Dronedaron, bei Patienten mit KHK Sotalol oder Dronedaron.

Das eigentliche Problem bei der Gabe von Antiarrhythmika sind Nebenwirkungen wie Torsades de Pointes. Mit dieser gefährlichen Nebenwirkung müsse insbesondere bei Klasse-I-Antiarrhythmika und Sotalol gerechnet werden. Dazu kommen substanzspezifische Nebenwirkungen. „Im direkten Vergleich mit Amiodaron ist Drone- daron zwar etwas weniger wirksam, dafür aber deutlich verträglicher", so Hohnloser. Dr. Peter Stiefelhagen

Satellitensymposium, Herz-Kreislauf-Tage 2013, Dresden, 25.1.2013 (Veranstalter: Sanofi-Aventis)

\section{Bessere $\mathrm{PCl}$-Ergebnisse durch FFR und OCT?}

_ Wird die Messung der fraktionellen Flussreserve (FFR) mit der optischen Kohärenztomografie (OCT) kombiniert, lassen sich bei Patienten mit stabiler oder akuter KHK perkutane Interventionen effektiver durchführen. Die optische Kohärenztomografie erlaubt eine Live-3D-Ansicht der Herzkranzgefäße und verbessert die Darstellung von Gefäßläsionen. Das neu zugelassene ILUMIEN ${ }^{\top M}$ OPTIS ${ }^{\mathrm{TM}} \mathrm{PCI}$ Optimization System ${ }^{\mathrm{TM}}$ bietet eine dreidimensionale $360^{\circ}$-Panoramadarstellung der Arterien in Echtzeit und damit ein vielseitiges Diagnosewerkzeug, das die exakte Platzierung von Stents unterstützt. Spezielle Software kombiniert dabei FFRMessung und OCT-Bildgebungstechnolo- gie in einem System. Durch die intravaskuläre Bildgebung können größere Gefäßbereiche nun in kürzerer Zeit realitätsnah dargestellt werden. Entscheidende Informationen zu Ort und Schweregrad der Koronararterienerkrankung sowie differenziertere Betrachtungsmöglichkeiten von Ischämien, Plaque-Ablagerungen oder Thromben sollen die medizinische Entscheidungsfindung erleichtern und dadurch auch zu besseren Behandlungsergebnissen und niedrigeren Kosten im Gesundheitswesen führen.

Dr. Ulrike Fortmüller

Symposium, EuroPCR-Kongress, Paris, 22.5.2013 (Veranstalter: St. Jude Medical) 